\title{
Full-Wave Intravascular Ultrasound Simulation from Histology ${ }^{\star}$
}

\author{
Silvan Kraft ${ }^{1,2}$, Sailesh Conjeti ${ }^{1}$, Peter B. Noël ${ }^{2}$, Stéphane Carlier ${ }^{3}$, \\ Nassir Navab ${ }^{1}$, and Amin Katouzian ${ }^{1}$ \\ 1 Computer Aided Medical Procedures, Technische Universität München, Germany \\ 2 Dept. of Radiology, Technische Universität München, Germany \\ 3 Dept. of Cardiology, UZ Brussel, Belgium
}

\begin{abstract}
In this paper, we introduce a framework for simulating intravascular ultrasound (IVUS) images and radiofrequency (RF) signals from histology image counterparts. We modeled the wave propagation through the Westervelt equation, which is solved explicitly with a finite differences scheme in polar coordinates by taking into account attenuation and non-linear effects. Our results demonstrate good correlation for textural and spectral information driven from simulated IVUS data in contrast to real data, acquired with single-element mechanically rotating $40 \mathrm{MHz}$ transducer, as ground truth.
\end{abstract}

\section{Introduction}

Simulating realistic ultrasound images for medical applications is still a challenging and open problem. One reason is that acoustic properties, such as refraction, diffraction, reflection, non-linearity and attenuation should be considered during simulation in accordance to the wave nature of ultrasound. The problem becomes even more complicated by the high heterogeneity of biological tissues as it is the case for atherosclerosis. Several approaches have been already proposed for simulating IVUS images. For example in [1], the Born approximation and a scatterer model were used and in [2] a ray-tracing algorithm on histopathology was deployed. Authors in [3] included Rayleigh scattering, physical reflection, transmission and absorption to realize a real-time simulation. In 4, a finite element model was combined with speckles incorporated by Field II.

With our proposed framework, we will introduce the first IVUS simulation that incorporates all effects of the wave propagation, including attenuation, multiple-scattering and non-linear effects based on a detailed physical model. This way the whole pipeline from the definition of the US transmitter up to the processing of the RF data leading to the final B-mode image are covered. With this framework we hope to achieve a better understanding of the tissuesound interactions and to be able to easily test setups with arbitraty hardware specifications, tissue-definitions and image processing algorithms.

^ This work was partially supported by the Bayerische Forschungsstiftung (project RoBildOR) and ACTIVE of the EU 7th Framework Program FP7/ICT-2009-6 under Grant Agreement No. 270460.

P. Golland et al. (Eds.): MICCAI 2014, Part II, LNCS 8674, pp. 627-634, 2014.

(C) Springer International Publishing Switzerland 2014 


\section{Methods}

\subsection{Solving the Wave Equation}

We chose the Westervelt equation to model the wave propagation and solved it with the finite differences time domain (FDTD) method [5]. One of the main advantages is that it describes the wave-propagation including thermo viscous diffusivity (attenuation) and non-linear effects. On the other hand, FDTD supports the use of heterogeneous grids in high resolution along with the parallelization of the code for graphics processing unit (GPU) implementation. The Westervelt-equation is defined as followed:

$$
\underbrace{\nabla^{2} p}_{1}-\underbrace{\frac{1}{c^{2}} \frac{\partial^{2} p}{\partial t^{2}}}_{2}+\underbrace{\frac{\delta}{c^{4}} \frac{\partial^{3} p}{\partial t^{3}}}_{3}+\underbrace{\frac{\beta}{\rho c^{4}} \frac{\partial^{2} p^{2}}{\partial t^{2}}}_{4}=0
$$

The first two terms $(1,2)$ correspond to the linear (lossless) wave equation with $p$ being the pressure, $c$ the speed of sound and $\partial t$ the time derivative. The third term (3) adds thermoviscous diffusivity with $\delta$ being the diffusivity coefficient. The diffusivity coefficient is related to the attenuation $\alpha$ as $\delta=2 \alpha \cdot \frac{c^{3}}{(2 \pi f)^{2}}(f$ being the center frequency). The last term (4) takes non-linearity effects into account with $\beta$ being the nonlinearity-coefficient and $\rho$ the local density.

Westervelt Equation in Polar Coordinates. As we are investigating an IVUS scenario with a rotating single-element transducer at the origin, the Westervelt-equation is solved in polar coordinates. Solving the equation in Cartesian coordinates was problematic concerning the precision of the rotation of the transducer within the grid. The inherit subpixel errors of the rotated transducer position led to inconsistent and noisy results concerning the final image, especially a smooth speckle pattern was not possible to achieve. Using polar coordinates greatly simplifies the rotation of the transducer as the rotation around the origin becomes a simple translational movement along the angle-direction, avoiding any positional errors.

The equation is solved with the explicit Euler scheme within a 2-dimensional Polar grid. Fourth order spatial derivatives and second order time derivatives were used as they yield a high enough precision at a good processing speed. In the following discretizations $n$ defines the current timestep, $r$ and $\phi$ the current radial and angular coordinates, $\Delta r, \Delta \phi$ and $\Delta t$ the corresponding discretization steps:

Laplace Operator

$$
\begin{aligned}
\nabla^{2} p \approx & \frac{1}{r} \frac{-p_{r+2}+8 p_{r+1}-8 p_{r-1}+p_{r-2}}{12 \Delta r} \\
& +\frac{-p_{r+2}+16 p_{r+1}-30 p_{r}+16 p_{r-1}-p_{r-2}}{12 \Delta r^{2}} \\
& +\frac{1}{r^{2}} \frac{-p_{\phi+2}+16 p_{\phi+1}-30 p_{\phi}+16 p_{\phi-1}-p_{\phi-2}}{12 \Delta \phi^{2}}
\end{aligned}
$$


Second time derivative - Second order central differences

$$
\frac{1}{c^{2}} \frac{\partial^{2} p}{\partial t^{2}} \approx \frac{1}{c^{2}} \frac{p_{n+1}-2 p_{n}+p_{n-1}}{\Delta t^{2}}
$$

Diffusivity Term

$$
\frac{\delta}{c^{4}} \frac{\partial^{3} p}{\partial t^{3}} \approx \frac{\delta}{c^{4}} \frac{p_{n+1}-3 p_{n}+3 p_{n-1}-p_{n-2}}{\Delta t^{3}}
$$

Non-linearity Term

$$
\begin{aligned}
\frac{\beta}{\rho c^{4}} \frac{\partial^{2} p^{2}}{\partial t^{2}} & \approx \frac{\beta}{\rho c^{4}} \cdot 2 \cdot\left(\left(\frac{p_{n}-p_{n-1}}{\Delta t}\right)^{2}+p_{n}\left(\frac{p_{n+1}-2 p_{n}-p_{n-1}}{\Delta t^{2}}\right)\right) \\
& =\frac{\beta}{\rho c^{4}} \frac{p_{n+1} p_{n}-p_{n}^{2}-p_{n} p_{n-1}+p_{n-1}^{2}}{\Delta t^{2}}
\end{aligned}
$$

With help of Equations (2)-(5) the Westervelt equation (11) is solved for the future timestep $n+1$.

Stability. It can be shown that the explicit solution becomes unconditionally unstable for high diffusivity-coefficients. However for high-frequency IVUS imaging of calcified plaques, high diffusivity needs to be applied. With the discretizations shown above a good compromise between precision, computing speed and appliable diffusivity was achieved when using a temporal resolution of $\Delta t=0.4 n s$ and a spatial resolution of $\Delta r=3.6 \mu \mathrm{m}$. In this case, the scheme is stable up to a maximum applied diffusivity of $\delta=3.5 \cdot 10^{-4} \frac{\mathrm{m}^{2}}{\mathrm{~s}}$.

Numerical Grid and Boundary Conditions. The choice of the polar-grid needs some consideration concerning the grid and its arrangement. First the singularity in the origin needs to be treated, here the gridpoints nearest to the origin were placed half a spatial step away from it to avoid the calculation of the singularity itself. Secondly a non-uniform angular resolution needs to be defined (See Figure 1) to avoid extremely dense gridpoints near the origin, which make very small timestep necessary. On the borders between the sections, the missing gridpoints were calculated by applying cubic interpolation. With a high spatial and temporal resolution the simulation of 256 scanline takes several hours on a GPU.

Concerning the boundary conditions we applied Dirichlet boundary conditions. The signal travels from the origin outwards, so the simulation will be finished before the reflected signal from the boundary will be received by the transducer. Absorbing boundaries, especially perfectly matched layers, are not necessary, instead they would increase the processing time with no benefit. 

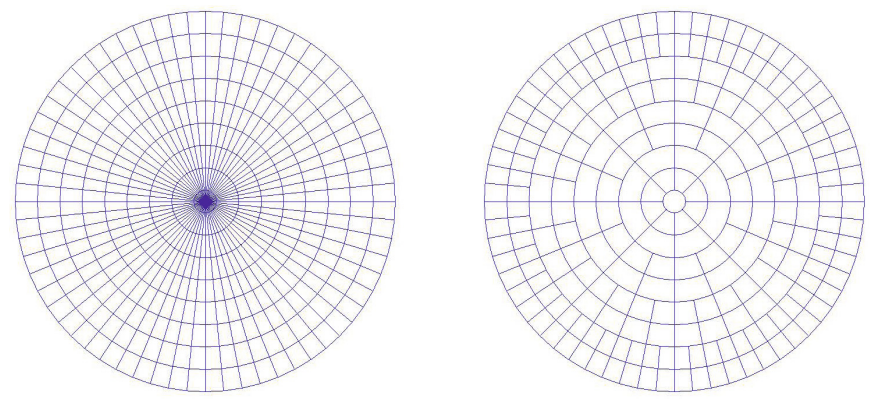

Fig. 1. Left: Polar grid with uniform angle resolution. Right: Polar grid with multiple sections of increasing angular resolution.

\subsection{Simulation Setup}

For the simulations within this work, a specific setup was chosen for which a histology slide and a real IVUS image for validation was available. The real image was made in vitro with saline replacing the blood within the vessel [6]. The simulation setup was appoximated as close as possible to the real setup in respect to the transducer design and the acoustic properties of the tissue.

Transducer Definition. We deployed Micro-CT to find out about the shape and the size of the active piezo-element (Fig. 2 left). In accordance to these findings, the virtual probe was defined with a width of $0.5 \mathrm{~mm}$ with no curvature. The virtual transducer element rotates with 256 scanlines per image. The excitation pulse was defined at $40 \mathrm{MHz}, 6$-cycle gaussian pulse, with a relative bandwidth of $50 \%$ (Fig. 2. right) and an amplitude of $500 \mathrm{kPa}$. The simulated beam characteristics shows a weakly focussed beam with a nominal resolution between 120-600 $\mu \mathrm{m}$ depending on the depth (Fig. 2, middle).
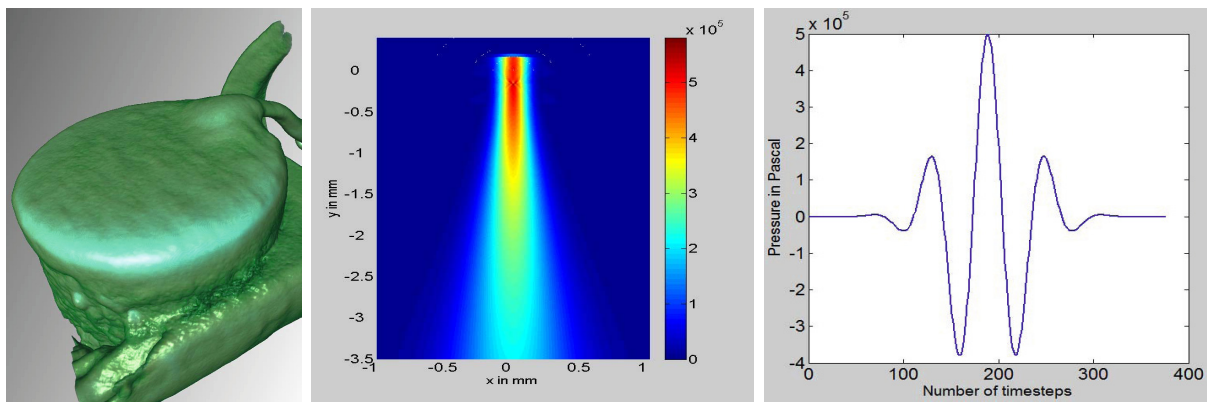

Fig. 2. Left: Rendered piezo-element of $40 \mathrm{MHz}$ single-element transducer (Atlantis, Boston Scientific). Middle: Simulated beam-characteristic (maximum pressure in $\mathrm{Pa}$ ). Right: Emitted ultrasound pulse used for simulation 
Phantom Generation. For creating the virtual phantom from the histology image, the histology was registered to the real US B-mode image with a non-rigid registration approach [7. In the following step, the deformed histology-image was manually labeled for different tissue types (lumen, calcified, lipidic, fibrotic, intima, media, adventitia and background tissue). For each of the tissue types certain acoustic properties were empirically defined (background speed of sound, diffusivity, nonlinearity, density).

Some of these parameters were measured and published in literature (8], [9], [10]), others had to be estimated. High attenuation for calcified areas was measured in [8] with $2.5 \mathrm{~dB} / \mathrm{mm} / \mathrm{MHz}$ and for thickened intima with $1.7 \mathrm{~dB} / \mathrm{mm} / \mathrm{MHz}$. This leads to high corresponding diffusivity coefficients for which the explicit numerical approach becomes unstable. The maximum diffusivity in calcified areas was set to $0.35 \cdot 10^{-3} \frac{\mathrm{m}^{2}}{\mathrm{~s}}$ for simulation. For saline the attenuation of $14 \mathrm{~dB} / \mathrm{cm}$ at $40 \mathrm{MHz}$ was reported [9] which corresponds to a diffusivity of $0.02 \cdot 10^{-3} \frac{\mathrm{m}^{2}}{\mathrm{~s}}$. The diffusivity of other tissue types was set to $0.08 \cdot 10^{-3} \frac{\mathrm{m}^{2}}{\mathrm{~s}}$.

Small heterogeneities (scatteres up to ten times smaller than the wavelength) are responsible for the strength of backscattered echoes in tissue and for the speckle patterns, these however havn't been measured experimentally yet. One main challenge in succeeding with the simulation of realistic ultrasound images is that there is no ground truth available concerning acoustic properties on a microscopic level. The only theoretical approach that defines scatterers in coronary arteries is based on the properties of nuclei in 11. Including nuclei alone lead to realistic speckle patterns but the scattering overall is too weak to be compared to real IVUS images.

To modell scatterers within the virtual phantom, for each tissue type one type of scatterer with a certain radius and a certain speed of sound difference to its background was defined. They are pseudorandomly distributed, each tissue is partitioned into cells of a fixed size and the scatterer is set on a random position within each of the cells. All applied parameters are presented within the following table 1. After defining the acoustic parameters, the phantom was transformed into polar coordinates with the nearest neighbour method.

Table 1. Acoustic properties used for simulation (SoS: Speed of sound, Sc.: Scatterer)

\begin{tabular}{|c|c|c|c|c|c|c|c|}
\hline Tissue Type & Saline & Calcified & Lipidic & Fibroti & Intima & Media & Adventitia \\
\hline Background SoS $\frac{m}{s}$ & 1590 & \multicolumn{5}{|c|}{1540} & 1600 \\
\hline Diffusivity in $\frac{m^{2}}{s}$ & $0.02 \cdot 10^{-3}$ & $0.35 \cdot 10^{-3}$ & $0.2 \cdot 10^{-3}$ & \multicolumn{4}{|c|}{$0.08 \cdot 10^{-3}$} \\
\hline Nonlinearity & \multicolumn{7}{|c|}{6} \\
\hline Density in $\frac{\mathrm{kg}}{\mathrm{m}^{3}}$ & 1049 & \multicolumn{6}{|c|}{1100} \\
\hline Sc. Cellsize in $\mu \mathrm{m}$ & - & 32 & \multicolumn{5}{|c|}{29} \\
\hline Sc. Diameter in $\mu \mathrm{m}$ & - & 25 & 22 & $\overline{7}$ & 7 & 11 & 14 \\
\hline Scatterer SoS $\frac{m}{s}$ & - & +1200 & -60 & +280 & +200 & +36 & +240 \\
\hline
\end{tabular}




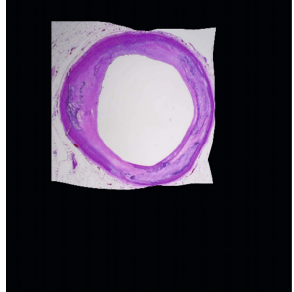

a.)

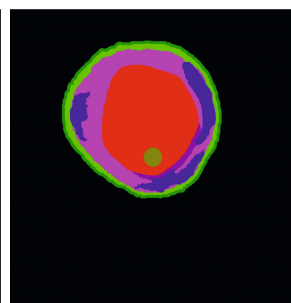

b.)

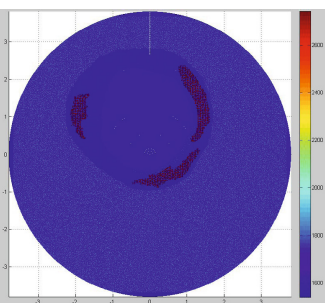

c.)

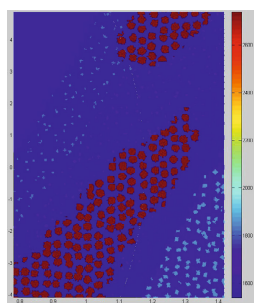

d.)

Fig. 3. (a) Registered histology image. (b) Labeled tissues (Red: Lumen, Pink: Intima, Blue: Calcification, Purple: Fibrosis, Green: Media, Darkgreen: Adventitia, Black: Background tissue, Olive: transducer position), (c) Speed of sound map, (d) Speed of sound map in detailed scatterer view

Post-Processing. For the RF-Data of the real IVUS images standard postprocessing steps were applied (i.e. band-pass filtering, envelope detection and log-compression). The same settings were used for the simulated RF-Data. Concerning the log-compression, the maximum and minimum reference-value was taken from the real RF-Data. This way the intensities within the B-mode images stay comparable.

\section{Simulation Results and Validation}

Qualitatively, the simulated results in Fig. 4 show a high correspondence with the real IVUS image. On a closer look, the backscattered intensitites of calcified regions are weaker in simulation as is the shadowing effect through attenuation behind the calcified areas. The first issue might result from non-optimal selection of scattering properties in lack of true values. The second issue concerning the shadowing is due to the constraints placed on the maximum value for the diffusivity for stability reasons. Also, the guide-wire wasn't included within the simulation, so the resulting artifact in the real image is not visible in the simulation.

The current pipeline for defining the virtual phantom, from dissecting the vessel, registrating the histology to the IVUS image and labeling it afterwards is feasible but comes with deviations. For this reason we decided subsequently to validate the simulated result with a maximum likelihood block matching motion estimation method, developed for noisy US images, namely $C D_{2}$ [12. Figure 5 (left and middle) shows a high similarity between simulation and real IVUS images for all tissue types. Lower similarity can be observed in the shadowing areas. The $C D_{2}$ performance measure was normalized to a $0-10$ scale with 10 indicating high-likelihood of consonance between matching blocks. For calcifications, the observed $C D_{2}$ value was $9.2 \pm 0.5$ with a minimum of 8.0. For simulation of fibrotic tissues, the $C D_{2}$ value was $9.5 \pm 0.3$ with a minimum of 8.7 . For intima, media and adventitia the values and the minima were $8.8 \pm 0.5$ (Min: 7.5 ), $8.7 \pm 0.6$ (Min: 7.1) and $8.6 \pm 0.6$ (Min:7.0). These consistent results are well 


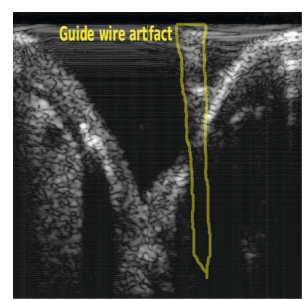

a.) Real IVUS

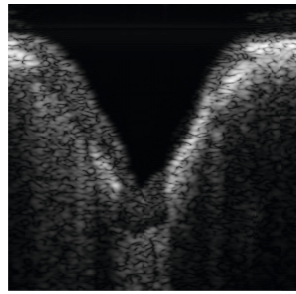

b.) Simulation

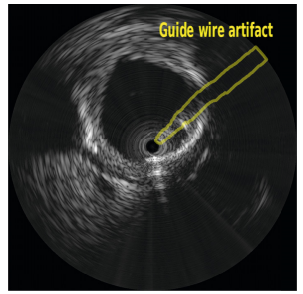

c.) Real IVUS

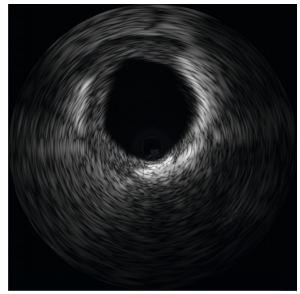

d.) Simulation

Fig. 4. a.) and b.) Comparison of B-mode images before scan-conversion and c.) and d.) after scan-conversion
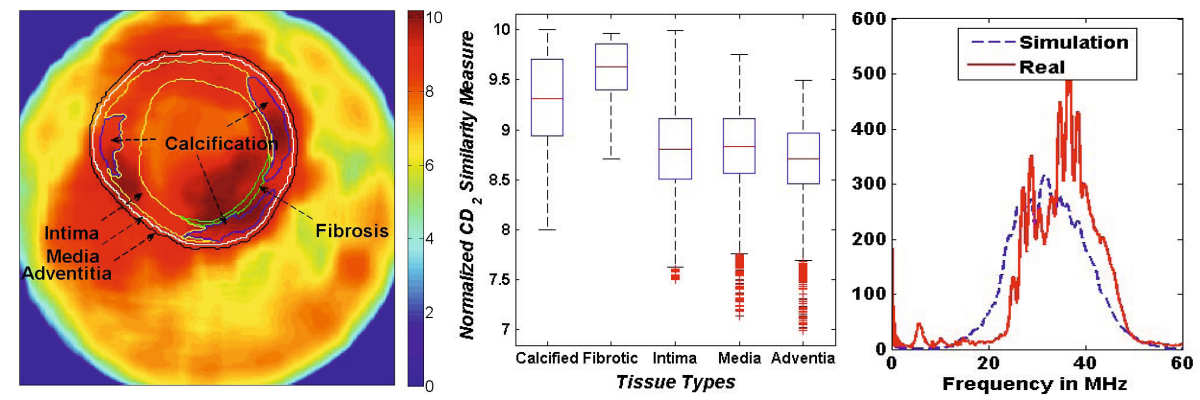

Fig. 5. Left: CD2 Similarity measure between real and simulated image. Middle: Normalized similarity measure (0-10) and standard deviation. Right: Frequency power spectrum of real and simulated images

within acceptable ranges and are strongly suggesting near-realistic performance of the simulated IVUS image.

Comparing the frequency spectra to each other (Fig. 5, right), they have a very similar range, which suggests that the bandwidth of $50 \%$ of the transducer is well chosen. The frequency shift can be attributed to typical fabrication-dependent variations of the mean frequency of IVUS probes.

\section{Conclusion}

In this work we introduce a framework that is capable of simulating physically realistic IVUS RF-data in $2 \mathrm{D}$, using histological priors with robust modelling of attenuation and non-linearity effects. For best results a solution in polar coordinates with a high spatial resolution was applied. This approach accounts for heterogeneity of atherosclerotic tissue. The bottelneck for achieving full correspondence with real IVUS images are the registration error and the missing insight on the acoustic properties and scatterer distributions within the tissue on a microscopic scale. Because of the high research interest in atherosclerosis and recent developments in acoustic microscopy, these missing data might be 
available in the near future. This framework will also be suitable for extended sensitivity analysis when varying specific parameters. One shortcoming of the proposed approach is the instability for high diffusivity values, which result in a weaker shadowing effect compared to real IVUS images. This could be solved by implicit approaches in trade for longer computing times. In future work we will investigate the influence of different hardware configurations on the IVUS image quality and after validation of the simulation for non-linear effects we plan to extend it for feasibility studies of intravascular harmonic imaging.

\section{References}

1. Ramírez, M.D.R., Ivanova, P.R., Mauri, J., Pujol, O.: Simulation model of intravascular ultrasound images. In: Barillot, C., Haynor, D.R., Hellier, P. (eds.) MICCAI 2004. LNCS, vol. 3217, pp. 200-207. Springer, Heidelberg (2004)

2. Groot, S.C., Hamers, R., Post, F.H., Botha, C.P., Bruining, N., Mc, E.: IVUS Simulation Based on Histopathology. Computers in Cardiology, 681-684 (2006)

3. Abkai, C., Becherer, N., Hesser, J., Männer, R.: Real-time simulator for intravascular ultrasound (IVUS). In: Proc. SPIE, vol. 6513, pp. 65131E-65131E-11 (2007)

4. Cardoso, F.M., Moraes, M.C., Furuie, S.S.: Realistic IVUS image generation in different intraluminal pressures. Ultrasound in Medicine \& Biology 38(12), 21042119 (2012)

5. Pinton, G.F., Dahl, J., Rosenzweig, S., Trahey, G.E.: A heterogeneous nonlinear attenuating full-wave model of ultrasound. IEEE Transactions on UFFC 56(3), 474-488 (2009)

6. Katouzian, A., Sathyanarayana, S., Li, W., Thomas, T., Carlier, S.G.: Challenges in tissue characterization from backscattered intravascular ultrasound signals. In: Proc. SPIE, vol. 6513, pp. 65130-11 (2007)

7. Katouzian, A., Karamalis, A., Lisauskas, J., Eslami, A., Navab, N.: IVUS-histology image registration. In: Dawant, B.M., Christensen, G.E., Fitzpatrick, J.M., Rueckert, D. (eds.) WBIR 2012. LNCS, vol. 7359, pp. 141-149. Springer, Heidelberg (2012)

8. Saijo, Y., Sasaki, H., Okawai, H., Nitta, S.I., Tanaka, M.: Acoustic Properties of Atherosclerosis of Human Aorta Obtained with High-frequency Ultrasound. Ultrasound in Medicine \& Biology 24(7), 1061-1064 (1998)

9. Treeby, B.E., Zhang, E.Z., Thomas, A.S., Cox, B.T.: Measurement of the ultrasound attenuation and dispersion in whole human blood and its components from 0-70 MHz. Ultrasound in Medicine \& Biology 37(2), 289-300 (2011)

10. Saijo, Y., Filho, E.S., Sasaki, H., Yambe, T., Tanaka, M.: Ultrasonic Tissue Characterization of Atherosclerosis by a Speed-of-Sound Microscanning System. IEEE Transactions on UFFC 54(8), 1571-1577 (2007)

11. Kraft, S., Karamalis, A., Sheet, D., Drecoll, E., Rummeny, E.J., Navab, N., Noël, P.B., Katouzian, A.: Introducing nuclei scatterer patterns into histology based intravascular ultrasound simulation framework. In: Proceedings of SPIE, vol. 8675, pp. 86750Y-6 (2013)

12. Cohen, B., Dinstein, I.: Motion estimation in noisy ultrasound images by maximum likelihood. In: Proceedings of the 15th International Conference on Pattern Recognition, vol. 3, pp. 182-185. IEEE Comput. Soc. (2000) 